\title{
Strengthening of a Polymer Interface: Interdiffusion and Crosslinking
}

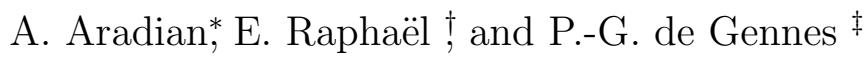 \\ Collège de France $\$ 11$ place Marcelin Berthelot \\ 75231 Paris Cedex 05, France
}

October 29, 2018

\begin{abstract}
In many industrial processes, pieces of the same polymer material are brought into contact at a temperature above the glass transition. Interdiffusion takes place across the interface and leads to a strengthening of the junction. Often, a crosslinker agent is also added in order to improve the global mechanical properties of the material, as in the formation of latex films from dispersed solutions of polymer particles. We studied theoretically the competition between the interdiffusion and the crosslinking reaction, and found that the control parameter tuning the balance between these two processes is $\alpha=Q \tau_{0} A_{0}^{*} N^{3} b^{3} / N_{e}$, where $Q \tau_{0}$ accounts for the reactivity of the crosslinker, $A_{0}^{*}$ is the initial concentration of sites capable of crosslinking on the polymer chains, $N$ is the polymerization index, $N_{e}$ the number of segments between entanglements and $b$ a distance comparable to the segment length. The case of practical interest is $\alpha \ll 1$ : the reaction locks the interfacial chains once a significant mixing has developed, resulting in films with good mechanical properties.
\end{abstract}

\section{Introduction}

When two pieces of the same polymer material are brought into good contact at a temperature above their glass transition temperature, the macroscopic interface between the pieces progressively disappears, whereas the mechanical strength of the interface increases. This phenomenon, of great practical importance, is known as "polymer-polymer welding" or "crack healing", and has been widely studied, both experimentally [1] and theoretically [2, 3, 4, 5]. The crack healing is primarily due to the diffusion of the polymer chains from both sides across the interface.

\footnotetext{
*Achod.Aradian@college-de-france.fr

${ }^{\dagger}$ Elie.Raphael@college-de-france.fr

${ }^{\ddagger}$ Pierre-Gilles.deGennes@espci.fr

${ }^{\S}$ Laboratoire de Physique de la Matière Condensée, C.N.R.S. URA 792
} 
In many situations, a crosslinker agent is added into the material: this is notably the case in the technology of latex coatings, where individual latex particles from a polymer dispersion are cast onto a surface (see the review on the subject by M.A. Winnik in ref [6]). Upon drying, particles form contacts and progressively coalesce to give a continuous film, whose mechanical properties may be significantly improved by crosslinker addition [7, \&].

The use of a crosslinker, however, brings some difficulties: a fine balance between interdiffusion and crosslinking rates is required to obtain optimal film strength, because reaction and diffusion enter into competition. The interdiffusion is strongly sensitive to molecular weight, and slows down when the crosslinking reaction advances and the chains become more and more branched. Thus if the reaction rate is too fast, particles will mix only partially, to the disadvantage of film tenacity. As this issue is crucial to industrial applications, experimental studies have been carried out monitoring the evolution of the interface at a microscopic level [9, 10].

Our aim in the present article, is to try, from a simple analysis based on scaling laws, to extract the important parameters that control the final state of the interface, and attempt to find some guidelines in optimizing systems displaying both interdiffusion and crosslinking. We do not hope, given the complexity of real situations, to afford a set of quantitative predictions.

The article is organized as follows. In section 2, we present our model, and derive the governing equations of the problem. In section 3, which will be of main interest for practical applications, we exhibit a control parameter and present the results of the model. In section 4, after discussing some limitations of our approach, we try to relate a macroscopic quantity as the adhesion energy to our previous microscopic results, and finally, we briefly consider a few other systems used in film coating technology that take benefit of alternative strengthening strategies.

\section{Governing equations}

\subsection{Assumptions of the model}

Initially, at the onset of contact between polymer particles, the chains near the surface are "reflected" at the interfaces (see Figure 1). Because of the new volume made available to them after contact, these distorted conformations progressively relax towards the equilibrium, Gaussian conformations of chains in a melt, which maximize the entropy of the system. However, as shown in [2, 3], this process does not take place through a Fickian diffusion of the chains: because of topological entanglements, the chains are trapped in the reflected tubes, and the only way to explore the volume on the opposite side of the interface is through the motion of chain ends which open the path across the junction and progressively drag along the rest of the chain according to a reptation mechanism.

In our system, a crosslinking agent is also added into the polymer particles, prior to contact. 


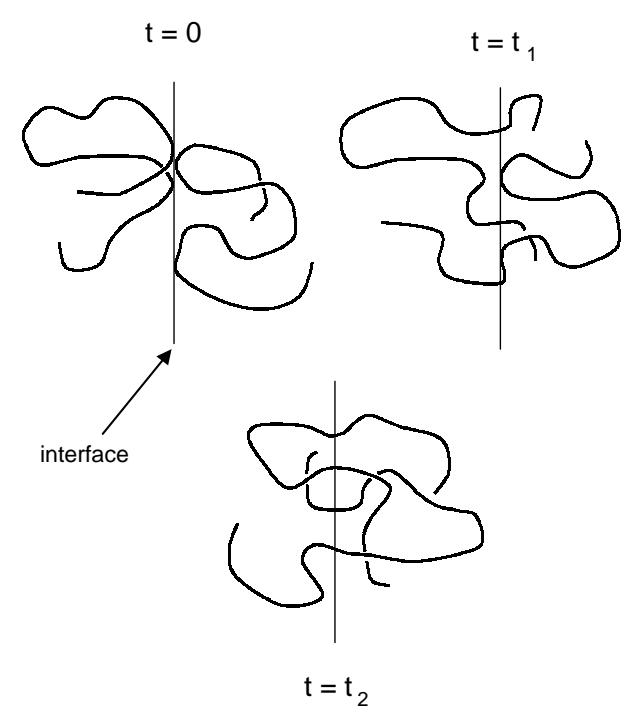

Figure 1: Aspect of interfacial chains at successive instants. Initially $(t=0)$, the chains are reflected at the interface, then chain ends start exploring the new volume across the border $\left(t=t_{1}\right)$, so that conformations progressively relax to the Gaussian, equilibrium shape $\left(t=t_{2}\right)$.

Hence, a chemical reaction proceeds in parallel to chain diffusion, and forms bridges between chains. This, in turn, has large consequences on the diffusion process itself, because crosslinked chains form branched objects whose motions are exponentially slower than linear chains [11]: interdiffusion dramatically slows down, as well as the reaction rate (which is related to chain motion). In the general case, providing a description of the subsequent evolution of the system is extremely complex, with a vast variety of macromolecules reacting one with the other (all with different numbers, positions and lengths of branches, and hence, different diffusion kinetics). Our description will thus rely on a few simplifying assumptions, which we are now going to present, deferring a critical discussion to section 4 .

We consider two latex particles of the same polymer, which are brought into contact at time $t=0$. The temperature is assumed to stay constant, and to exceed the glass transition temperature of the samples. The macromolecules, which we assume to be linear and monodisperse, consist of $N$ units ( $N$ taken greater than the entanglement threshold $N_{e}$ ), and are statistical copolymers of two types of monomers, $A$ and $A^{*}$. The crosslinker, called $X$, is a bi-functional agent, able to bind only to $A^{*}$ sites. The crosslinking reaction sets up in two steps. First, the crosslinker molecules $X$ attach to the "active" sites $A^{*}$, yielding $P A^{*}+X \rightarrow P A^{*}-X$ (where $P$ stands for "polymer backbone"). Then, in a second reaction, the "true" crosslinking between chains occurs, yielding $P A^{*}-X+A^{*} P \rightarrow P A^{*}-X-A^{*} P$. The first step involves the diffusion of a small molecule $X$ and should be very fast compared to the second step, which involves reaction 


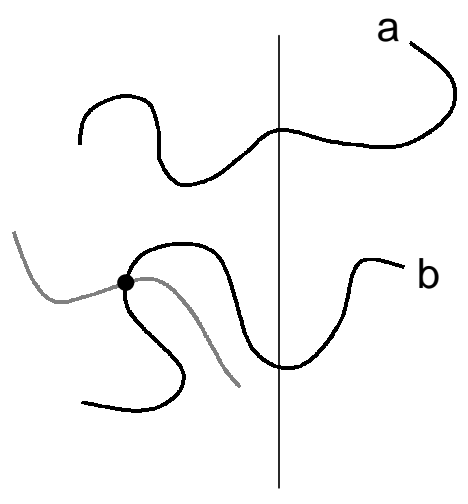

Figure 2: Different types of chains: Chain 'a' is mobile; chain 'b' is considered to be fixed, because it formed a crosslink (black dot) with another (grey) chain (the vertical line depicts the initial interface between the adjacent particles).

of sites both borne by polymer chains. For this reason, in the rest of the article, we will consider only the last step (polymer-polymer reaction), assuming that the attachment step is entirely completed at $t=0$ [12].

We also restrict ourselves to the case of only one $X$ attached per chain. This assumption is not meant solely to bring a simplification, but is also founded on practical grounds: from the classical work of Gent and coworkers [13, 14], we know that for elastomers, a tighter network, as resulting from an increased $X$ concentration, makes a poorer adhesive. Our hope is thus that this hypothesis still allows for most situations of interest.

Finally, we assume that when a chain binds to another, the resulting branched object remains fixed in position; this is in contrast with mobile chains, that did not undergo any reaction (except the binding of an $X$ molecule) and still keep their full mobility (see Figure 2). Of course, this assumption is not entirely realistic, because branched objects have a finite -though reducedmobility. We shall come back to this point at the end of the article.

\subsection{Extent of mixing $\Gamma_{f}$}

We add crosslinkers to enhance the mechanical strength of the overall film that is obtained after coalescence of the particles. At the microscopic scale of chains, the strengthening of an interface is due to the interdiffusion between the two blocks, i.e., the extent of mixing between adjacent particles. At a given time, if we consider the chains that have crossed the interface, some are fixed (because of prior crosslinking) so that their contribution to the mixing is permanent, and some are mobile and their contribution is bound to evolve. As the crosslinking reaction 
proceeds, the mobile chains "disappear" to the benefit of fixed ones, so that sooner or later, any chain that once contributed in a "transient" way to the extent of mixing finally contributes "permanently". Ultimately, all chains are fixed and the extent of mixing reaches a maximum value which characterizes the final state of the film. In the following, because it is of greater physical importance, we will focus our attention mainly on the permanent part of the extent of mixing, $\Gamma_{f}$ (taking only fixed chains into account), and will consider the transient part $\Gamma_{m}$ only incidentally.

As stated already, the motion of the chains is actually led by the motion of chain ends across the interface. Let us define $\rho_{0}$ as the initial $(t=0)$ density (per unit volume) of mobile chain ends at position $x$, and make the hypothesis that it is uniform (no segregation of chain ends at the interface). Denoting $x$ the abscissa on the axis perpendicular to the interface (which is located at $x=0)$, we also define $\rho_{m}(x, t)$ and $\rho_{f}(x, t)$ as, respectively, the densities of mobile chain ends and fixed chain ends at position $x$ and time $t$. Our first relation comes from the conservation of the total number of chain ends, yielding:

$$
\frac{d \rho_{m}}{d t}(x, t)=-\frac{d \rho_{f}}{d t}(x, t)
$$

The next point is to notice that, as far as the ongoing crosslinking reaction is concerned, the medium is spatially homogeneous. The argument proceeds as follows. At $t=0$, chains near the interface indeed start from out-of-equilibrium configurations. But, to first order, if we assume that the contact is perfect and that the melt density is the same at the interface and in the bulk, the diffusion modes of these distorted chains are the same as those of equilibrium bulk chains. As it is intimately related to these diffusion processes, the reaction rate must accordingly remain uniform in the system. In other words, this means that, wherever it is located, any given unit volume of the system hosts the same number of reactions.

In order to keep track of the reaction advancement, let us call $r(t)$ the 'reacted fraction', i.e., the fraction of the initially mobile chains whose $A^{*} X$ site has reacted between time $t=0$ and time $t$ (implying $r=0$ at $t=0$, and $r=1$ at the end of the reaction). Each time a chain reacts, it becomes a fixed branched object, so that $r(t)$ also gives the fraction of mobile chains that have become fixed by reaction, yielding [15:

$$
\rho_{m}(x, t)=\rho_{0}[1-r(t)] .
$$

From this, using eq 1, we deduce

$$
\rho_{f}(x, t)=-\int_{0}^{t} \frac{d \rho_{m}}{d t} d t=\rho_{f}(t=0)+\rho_{0} r(t),
$$

where $\rho_{f}(t=0)$ is the initial density of fixed chains (initial network). 


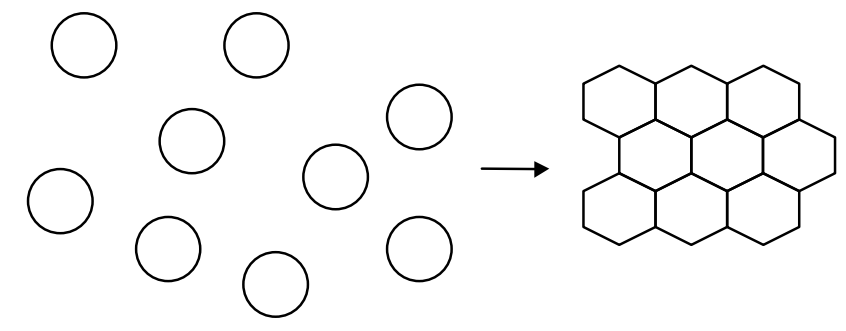

Figure 3: When an aqueous dispersion of spherical polymer particles dries (on the left), the particles enter into contact and are deformed into space-filling polyhedral cells (on the right). This is the initial $t=0$ situation of our model, where we consider only such flat interfaces. Neighboring cells then progressively coalesce to form a continuous film.

Let us describe the evolution of the interdiffusion for times smaller than the reptation time of the chains $T_{\text {rep }}$. At $t=0$, the film is made of the juxtaposition of polyhedral cells [6], displaying flat interfaces with their neighbors (Figure 3). Adjacent cells then start coalescing by interdiffusion. In a given cell 1 , we consider a thin slice of material, chosen parallel to the interface with cell 2 , and located at some position $x$ (see Figure 4). When do the first chain ends coming from cell 2 start to invade this slice? To answer this question, we have to know the distance traveled by a chain end in a given time $t$. According to the reptation theory 16, 17], each chain diffuses inside a tube, and over a time $t$, travels a (r.m.s.) tube length given by $s(t)=a\left(N / \sqrt{N_{e}}\right)\left(t / T_{r e p}\right)^{1 / 2}$, where $a$ denotes the length of a chain segment, and $T_{r e p}=N^{3} \tau_{0} / N_{e}$ is the reptation time of the chain ( $\tau_{0}$ the relaxation time of a monomer). To this curvilinear length corresponds a distance "as the crow flies", $l_{r e p}(t)$, which is given by:

$$
l_{\text {rep }}(t)=\left(s a \sqrt{N_{e}}\right)^{1 / 2}=R_{0}\left(\frac{t}{T_{\text {rep }}}\right)^{1 / 4} \quad\left(t \lesssim T_{r e p}\right),
$$

where $R_{0}=a N^{1 / 2}$ is the Gaussian extension of a chain. Within constants, this distance $l_{\text {rep }}(t)$ is also the (rectilinear) distance traveled by the chain ends. Inverting this formula, we can now see that the first chain ends from cell 2 reach the slice at $x$ in cell 1 at a time

$$
t_{x}=T_{r e p}\left(\frac{x}{R_{0}}\right)^{4}
$$

We now want to compute $\Gamma_{f}$, the extent of mixing due to permanent chains. All chains passing through the slice do not pursue their migration, a fraction of them is stopped there by crosslinking. This situation is accounted for by the local augmentation of $\rho_{f}$. From $t=0$, the density of crossing chain ends $\rho_{f}^{\text {cross }}$ (from cell 2) that have settled at $x$ (in cell 1 ) may then be 


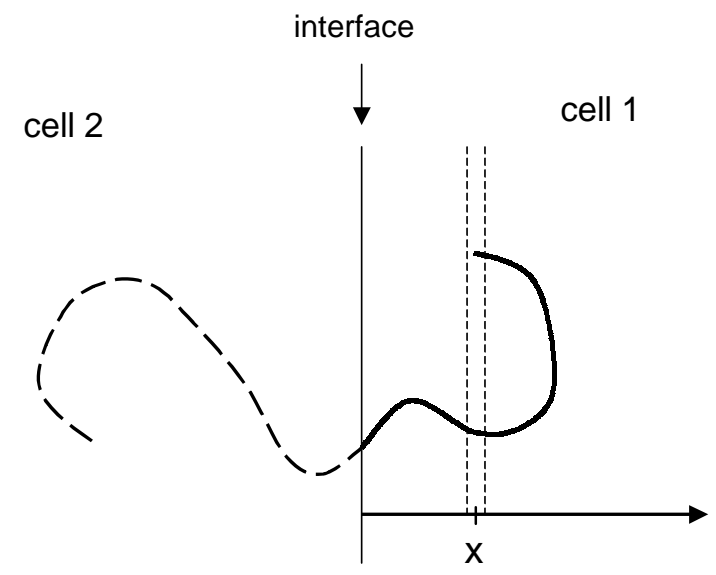

Figure 4: A chain from cell 2 invading cell 1, with its end located in a slice at a distance $x$ from the interface. The number of monomers in the "invading portion" of the chain (solid line) can be estimated to $\simeq x^{2} / a^{2}$. The monomers in the dashed part of the chain should not enter into the computation of the extent of mixing.

written as

$$
\rho_{f}^{\text {cross }}(x, t)= \begin{cases}\rho_{f}(t)-\rho_{f}\left(t_{x}\right) & \text { if } t>t_{x} \\ 0 & \text { if } t \leq t_{x}\end{cases}
$$

(where we took into account that chain ends settling down at times earlier than $t_{x}$ do not come from cell 2). Of course, eq 6 is not exact, and oversimplifies the density profiles generated by the diffusion process, but it should retain the main features.

Counting that each invading chain end located at $x$ drags along approximately $x^{2} / a^{2}$ monomers, because dragged chains form random walks (see Figure 4), and summing over all possible slices, we are now able to give a formula for the extent of mixing $\Gamma_{f}$ :

$$
\Gamma_{f}(t)=\int_{0}^{\infty} \rho_{f}^{\text {cross }}(x, t) \frac{x^{2}}{a^{2}} d x \quad\left(t \lesssim T_{\text {rep }}\right) .
$$

Finally, with the use of $\rho_{f}^{\text {cross }}$ (eq 6) and $\rho_{f}$ (eq 3 ), we arrive at

$$
\Gamma_{f}(t)=\frac{\rho_{0}}{a^{2}}\left(r(t) l_{\text {rep }}^{3}(t)-\int_{0}^{l_{r e p}(t)} r\left(t_{x}\right) x^{2} d x\right) \quad\left(t \lesssim T_{\text {rep }}\right)
$$

(ignoring numerical factors, which would not be very meaningful at our scaling law level).

We can also calculate the contribution $\Gamma_{m}$ from mobile chains. In this case, with obvious notations, we have $\rho_{m}^{\text {cross }}(x, t)=\rho_{m}(t)-\rho_{m}\left(t_{x}\right)$ if $t>t_{x}$, and $\rho_{m}^{\text {cross }}(x, t)=0$ otherwise, and 


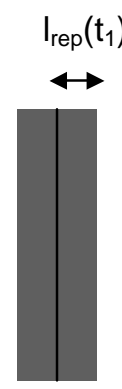

(a)

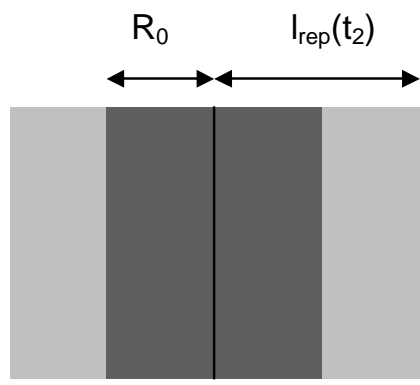

(b)

Figure 5: Extent of mixing between two cells, at two different times $t_{1}$ and $t_{2}$ (the vertical, black, line depicts the interface). (a) At time $t_{1}<T_{\text {rep }}$, the mixing occurs inside the shaded region of breadth $l_{\text {rep }}\left(t_{1}\right)$. (b) At time $t_{2}>T_{\text {rep }}$, the mixing has increased and takes place over a region of width $l_{\text {rep }}\left(t_{2}\right)$ (dark and light regions). However, only the chains located inside the dark region of width $R_{0}$ effectively bridge the interface: from the point of view of interface strength, this is the "efficient" part of the mixing.

thus

$$
\Gamma_{m}(t)=\int_{0}^{\infty} \rho_{m}^{\text {cross }}(x, t) \frac{x^{2}}{a^{2}} d x=\frac{\rho_{0}}{a^{2}}[1-r(t)] l_{r e p}^{3}(t) \quad\left(t \lesssim T_{r e p}\right) .
$$

We now turn to the evolution of the system after the reptation time $T_{r e p}$. Chains from each side of the interface keep diffusing further into the cell on the other side, so that the mixing between adjacent cells keeps increasing. However, for time $t>T_{\text {rep }}$, the traveled distance $l_{\text {rep }}(t)$ becomes greater than the Gaussian radius of the chains $R_{0}$. Thus, some chains migrate farther than a distance $R_{0}$ from the interface and lose any intersection with it, so that they do not bridge the junction anymore: although they improve the mixing between cells, such chains do not contribute to the strength of the interface. Since our interest lies in keeping track of the strengthening of the interface, rather than of the mixing by itself, we must discard these leaving chains from our computation, and keep only the "efficient" part of $\Gamma_{f}$ and $\Gamma_{m}$ (see Figure 5). This is done by saturating the length $l_{r e p}$ to $R_{0}$ after time $T_{r e p}$ in equations 869 . The modified equations at times greater than $T_{r e p}$ take the form:

$$
\Gamma_{f}(t)=\frac{\rho_{0} R_{0}^{3}}{a^{2}} r(t)-\frac{\rho_{0} R_{0}^{3}}{a^{2}} \int_{0}^{R_{0}} r\left(t_{x}\right) x^{2} d x \quad\left(t \gtrsim T_{r e p}\right),
$$

and

$$
\Gamma_{m}(t)=\frac{\rho_{0} R_{0}^{3}}{a^{2}}[1-r(t)] \quad\left(t \gtrsim T_{r e p}\right) .
$$


We notice that, in eq 10, the second term containing the integral is now a constant independent of time.

\section{$2.3 \quad$ Reacted fraction $\mathrm{r}(\mathrm{t})$}

In section 2.2, the reacted fraction $r(t)$ was defined as the fraction of the mobile chains present initially whose $A^{*} X$ site has reacted with another site between $t=0$ and time $t$. Initially, at $t=0$, all the $A^{*} X$ sites are unreacted, yielding $r=0$. At the end of the reaction, all the $A^{*} X$ have reacted and $r=1$. To complete our calculation of $\Gamma_{f}$ and $\Gamma_{m}$, we now need to compute the reacted fraction $r(t)$.

Because of the non-Fickian laws for segmental diffusion in polymeric materials, reaction kinetics may display very special features in the so-called 'diffusion-controlled regime' [18]. However, for reagents of common reactivity, the practical case is usually the 'mean-field regime' [19], that is to say, the regime of classical, small molecules, reaction kinetics, and this is indeed the regime that will be assumed in the following.

We start by defining $Q$ as the 'local' reactivity of the active sites $A^{*} X$, to be understood as the probability of reaction per unit time of a pair $A^{*} X / A^{*}$ provided they are in permanent contact. Then the reaction constant $k$ is 18,20 ,

$$
k=Q b^{3},
$$

where $b$ is the capture radius, i.e., the distance below which reaction becomes possible. This capture radius is a molecular length, and we will often simply assume $b \simeq a$ (chain unit size).

We define $X_{0}$ as the initial $(t=0)$ concentration of $A^{*}$ sites bound to an $X$ molecule, i.e., the initial concentration in $A^{*} X$ groups. $A_{0}^{*}$ denotes the initial concentration in the remaining $A^{*}$ sites, i.e., not bound to any $X$. Initially, the concentration of intermolecular bridges $A^{*} X A^{*}$ is zero. We also define a parameter $\eta=X_{0} / A_{0}^{*}$. In our assumptions, there is only one $X$ attached per chain, but $A^{*}$ sites may be more numerous, so that we necessarily have $\eta \leq 1$. A straightforward calculation of chemical kinetics yields the number of $A^{*} X$ sites that have been consumed by the bridging reaction $A^{*} X+A^{*} \rightarrow A^{*} X A^{*}$. Then, to compute the desired fraction $r(t)$ from this number, we simply need to divide it by the initial concentration $X_{0}$.

For $\eta<1$, we obtain

$$
r(t)=\frac{1-\exp \left[-(1-\eta) A_{0}^{*} Q b^{3} t\right]}{1-\eta \exp \left[-(1-\eta) A_{0}^{*} Q b^{3} t\right]} \quad(\eta<1),
$$

which can be easily rearranged to introduce the reptation time $T_{r e p}=N^{3} \tau_{0} / N_{e}$ into

$$
r(t)=\frac{1-\exp \left[-(1-\eta) Q \tau_{0} \mu\left(t / T_{r e p}\right)\right]}{1-\eta \exp \left[-(1-\eta) Q \tau_{0} \mu\left(t / T_{r e p}\right)\right]} \quad(\eta<1),
$$


where a new important quantity appears: $\mu \equiv A_{0}^{*} N^{3} b^{3} / N_{e}$.

For the marginal case $\eta=1$, a similar calculation can be performed, and yields

$$
r(t)=\frac{Q \tau_{0} \mu\left(t / T_{r e p}\right)}{1+Q \tau_{0} \mu\left(t / T_{r e p}\right)} \quad(\eta=1) .
$$

\section{Results}

We have seen in the previous sections, that when two particles come into contact, the interface strengthens because of the interdiffusion and the crosslinking of chains at the junction. At a microscopic level, the extent of mixing between the adjacent particles $\Gamma_{f}$, due to fixed (crosslinked) chains, provides a good account of this strengthening. We are now able to give results for this extent of mixing, using eqs 811 for the extent of mixing and eqs 14,15 for the reacted fraction $r(t)$.

We will split our discussion into two cases, depending on the relative rates of the crosslinking reaction and of the interdiffusion process. As we shall see, the crucial control parameter in the system is

$$
\alpha=Q \tau_{0} \mu=Q \tau_{0} A_{0}^{*} \frac{N^{3}}{N_{e}} b^{3}
$$

In eq 16, $Q \tau_{0}$ is a dimensionless number, corresponding to the probability of reaction for one collision of reactive sites $\left(Q\right.$ is the probability per unit time, and $\tau_{0}$, the monomer relaxation time, is similar to the collision duration).

\section{1 'Fast reaction' regime: $\alpha \gg 1$}

We start with the case $\alpha \gg 1$. If we consider the reaction rate $\mathrm{r}(\mathrm{t})$ (eq 14), we easily see that $r(t) \simeq 1$ is reached (end of reaction) for $t \gg \frac{T_{r e p}}{(1-\eta) \alpha}$, i.e., at times much smaller than $T_{\text {rep }}$ since $\alpha \gg 1$. (We stress that here $\eta$ cannot be chosen too close to 1 , or we must use another expression for $r(t))$. Evaluating $\Gamma_{f}(\mathrm{eq} 8)$ at times great enough so that the reaction can safely be considered as complete, we find that the final value is:

$$
\Gamma_{f}^{f i n a l} \simeq \frac{\rho_{0} R_{0}^{3}}{a^{2}} \frac{1}{[(1-\eta) \alpha]^{3 / 4}}
$$

(within prefactors of order unity), which, as will be seen shortly, is a very small value compared to the maximum reachable extent of mixing $\Gamma_{f}^{\max } \simeq \rho_{0} R_{0}^{3} / a^{2}$.

We should also mention the marginal case, when $\eta$ is chosen very close to 1 . Then the correct expression for $r(t)$ is given by eq 15, but $\Gamma_{f}^{f i n a l}$ remains very similar (and very small):

$$
\Gamma_{f}^{f i n a l} \simeq \frac{\rho_{0} R_{0}^{3}}{a^{2}} \frac{1}{\alpha^{3 / 4}}
$$


(within prefactors).

We conclude that this situation is not favorable to the development of a strong junction at the particles' interfaces, and should be avoided. Actually, in this case, the reaction is so fast compared to the interdiffusion, that all chains are fixed by crosslinking well before they could bridge the interface efficiently.

\section{2 'Slow reaction' regime: $\alpha \ll 1$}

We now take $\alpha \ll 1$. Here the reaction is complete at times $t \gg T_{r e p} / \alpha$ which are larger than $T_{\text {rep }}$. To evaluate $\Gamma_{f}^{f i n a l}$, we shall then use the form valid at times greater than $T_{\text {rep }}$, as given by eq 10. In this expression, one can actually neglect the contribution from the integral, with respect to the first term, because for $x \leq R_{0}, r\left(t_{x}\right) \ll 1$. The extent of mixing $\Gamma_{f}$ hence evolves as $r(t) \rho_{0} R_{0}^{3} / a^{2}$, and reaches a final value

$$
\Gamma_{f}^{\text {final }} \simeq \frac{\rho_{0} R_{0}^{3}}{a^{2}}
$$

which is the largest physically realizable value in our system.

We note that, if we have $\eta \simeq 1$, using the appropriate expression of $r(t)$ yields the same result.

This 'slow reaction' regime is thus the interesting one for practical purposes, because one allows the interdiffusion to develop fully before locking the formed bridges in position. We may also add that, in practice, $\alpha$ will probably not need to be much smaller than unity for the system to display a good strengthening.

One might wonder whether $\alpha \ll 1$ is an easy value to find in real systems. If we exclude highly reactive groups, like radicals, usually $Q \tau_{0}$ is well below $10^{-6}$ [21]. The concentration of $A^{*}$ sites also plays an important role. If there are a few $A^{*}$ sites per chain, $A_{0}^{*} \simeq 1 / N a^{3}$, then $\alpha \simeq Q \tau_{0}\left(N^{2} / N_{e}\right)(b / a)^{3}$, which is around $10^{-4}$ with $Q \tau_{0}=10^{-8}, N=10^{3}, N_{e}=10^{2}$ and $b \simeq a$. With many $A^{*}$ on each chain, say $A_{0}^{*} \simeq p / a^{3}$ ( $p<1$ but not too small), we get $\alpha \simeq Q \tau_{0}\left(N^{3} / N_{e}\right) p$, which can still be made small: $Q \tau_{0}=10^{-8}, N=10^{3}, N_{e}=10^{2}, p=0.1$, yield $\alpha=0.01$.

\subsection{A simple interpretation of $\alpha$}

We will here provide a simple explanation of the control parameter $\alpha=Q \tau_{0} A_{0}^{*} N^{3} b^{3} / N_{e}$ that has been exhibited above. For this purpose, let us consider an $A^{*} X$ group located on a given chain chosen at random among the others, and follow its evolution from the beginning of the crosslinking reaction, at $t=0$.

Thinking in terms of a lattice model, where the $A^{*} X$ molecule moves with a jump frequency $1 / \tau_{0}$ (where $\tau_{0}$ is the monomer relaxation time), we may say that the $A^{*} X$ group makes $T_{r e p} / \tau_{0}$ 
displacements from $t=0$ until $t=T_{r e p}$. At each of these movements, the $A^{*} X$ molecule may react and form a crosslink with any of the $A^{*}$ molecules lying within a capture volume $b^{3}$. In our mean-field situation, we can estimate the number of such candidates approximately as $A_{0}^{*} b^{3}$. However, only a fraction of these collisions with the possible candidates bring an effective reaction: with $Q$ the 'local' reactivity, i.e., the probability of effective reaction per unit time when an $A^{*} X$ encounters an $A^{*}$, and $\tau_{0}$ the contact time, we find that an $A^{*} X$ molecule may have $Q \tau_{0} A_{0}^{*} b^{3}$ effective crosslinking reactions at each displacement. (Note that this is only a virtual number of reactions, since an $A^{*} X$ site is able to react only once.) Hence, over a time $T_{\text {rep }}$, we deduce that an $A^{*} X$ group (virtually) makes $Q \tau_{0} A_{0}^{*} b^{3} T_{r e p} / \tau_{0}$ reactions. Noticing that $T_{\text {rep }}=N^{3} \tau_{0} / N_{e}$, we see that this quantity is the same as $\alpha$. Thus, $\alpha$ can be understood as the number of crosslinks that a given chain may form by reaction of its $A^{*} X$ site, if it were able to react an infinite number of times.

We can now go further and understand the fast and slow reaction regimes in terms of characteristic times. On one hand, we define a characteristic crosslinking time as the time required for an $A^{*} X$ site to react (once) with an $A^{*}$ molecule. Since an $A^{*} X$ site may have $\alpha$ (virtual) reactions in a time $T_{r e p}$, we estimate this characteristic time for one reaction as $T_{r e p} / \alpha$. On the other hand, the characteristic time for the interdiffusion process is $T_{\text {rep }}$, i.e., the time needed for an interfacial chain to relax entirely from its initial contorted conformation.

Comparing these characteristic times together, we are able to retrieve, in a simple way, some conclusions that have been exposed previously. When $\alpha \gg 1$ (i.e., $T_{r e p} / \alpha \ll T_{\text {rep }}$ ), the reaction is faster than the interdiffusion, locking the chains before they can bridge the interface. On the opposite, when $\alpha \ll 1$ (i.e., $T_{\text {rep }} / \alpha \gg T_{r e p}$ ), the reaction mainly occurs after an appreciable amount of interdiffusion has set up, and enhances the mechanical properties of the film.

\section{Discussion}

\subsection{Further remarks}

The approach that has been presented here is rather simplified, and could be improved in several ways. We list below a few points that would need to be worked on in order to get a more accurate description of the evolution of the system, that would go farther than scaling laws.

i) We restricted ourselves to monodisperse systems, but practical situations may prove more difficult. One of the effects of polydispersity is to give rise to a range of chain diffusion coefficients, and hence to a blurring of simple scaling laws as the ones that were used here (this has already been observed on systems displaying interdiffusion alone [22]). Other effects of polydispersity

are discussed in Taylor and Winnik [7]. Another point to notice is that, in real systems, having precisely one $X$ molecule bound per polymer chain is unfeasible: the number of $X$ per chain 
will rather display a statistical distribution of possible values, with an average of order unity. This distribution may also present spatial variations inside each particle: in the example of latex films, before contact between individual particles is set, the $X$ crosslinker molecules are originally outside the particles, in the aqueous solution, and progressively diffuse inside. The concentration profile arising from such a diffusion process will inevitably present spatial dependences.

ii) As pointed out in our model assumptions, we consider that branched objects remain fixed. This is not completely true, and one may imagine that in the late stages of the reaction most unreacted sites are borne by arms belonging to branched molecules. A complete theory would thus certainly need to take the kinetics resulting from reactions between such molecules into account. Very slow evolutions were indeed observed in experiments on certain types of latex films 23].

iii) Another assumption of our approach is that the kinetics of polymer reactions remains the same as that of small molecules, due to the weak reactivity of usual chemical species. However, as was shown by O'Shaughnessy in [20], there is necessarily a crossover at long times, from the usual kinetics regime to the 'diffusion-controlled' (DC) regime resulting from the non-Fickian diffusion of segments (the so-called 'compact exploration'). We checked that, with usual chain lengths and usual reactivities of crosslinking sites, the crossover would take place well after completion of the crosslinking reaction. Would the DC regime set up earlier, for example if we choose very reactive agents $\left(Q \tau_{0} \simeq 1\right)$, this would be harmful to the mechanical properties of the interface, and thus an undesirable situation.

iv) A last question is related to the surface state of the particles before they start merging together. In order to stabilize the initial solution, surface-active agents may be present. After onset of contacts, they might modify the diffusion kinetics of the chains across the interfaces. Also, with certain polymer compositions, the particles themselves may have a 'core-shell' structure, where the outer part of the particles display distinct properties compared to the inside.

\subsection{Estimation of the adhesion energy}

From a macroscopic point of view, the strengthening of the interfaces may be characterized by several physical quantities. One of them is the adhesion energy, defined as the work required to separate two particles by opening a fracture at the interface. We will here present an attempt to estimate this energy, in the limit of very low separation velocities.

We start with the fast reaction case $\alpha \gg 1$ (section 3.1), and consider the final state, after completion of the reaction. Most of the chains were stopped by reaction very early in the process of interdiffusion. If we look at chains originally from the left side of the interface, we notice that these reactions most probably occurred on the longest part of the chain, i.e., the portion still in the original particle on the left. If we now try to separate the two adjacent particles, we 


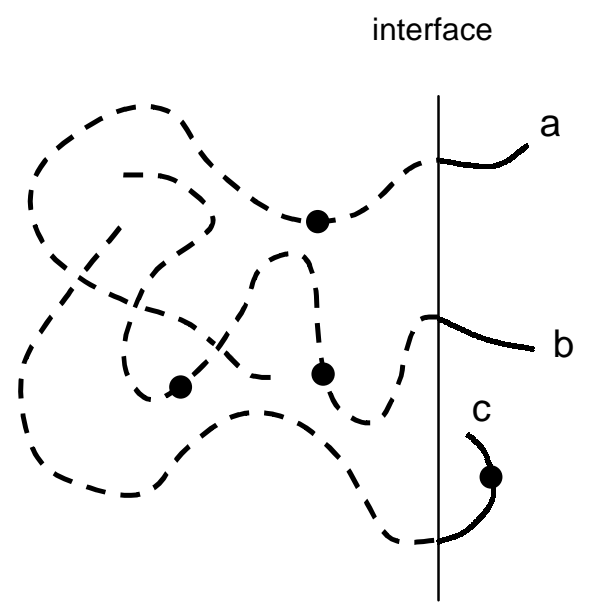

Figure 6: Chains at the interface in the final state of the fast reaction regime. Chains 'a' and 'b' depict the most common case, where the crosslinks (black dots) occurred on the portions which remained in their home particle (dotted lines). For chain 'c', the reaction occurred in the portion inside the other particle, but this a rare event (shortest arm). When a fracture opens, most chains (like 'a' and 'b') will pull the portions in solid lines out.

will have to pull out the portions of chains that have penetrated into the right-hand particle (see Figure 6). Some years ago, Raphaël and de Gennes developed a model [24] evaluating the adhesion energy when one pulls 'connector' chains grafted on a surface out of an elastomer. It was found that the adhesion energy $G$ in the quasi-static limit (very slow separation) is given by

$$
G=U_{v} \sigma n
$$

where $U_{v}$ is a Van der Waals energy, $\sigma$ the number of connectors per unit area of interface, and $n$ the polymerization index of the connectors. If we try to extend this expression to our situation, we must integrate eq 20 over a distribution of connector lengths, since the effective pull-out length that has to be counted is the (varying) portion inserted into the adjacent particle (and not the total chain length $N$ ). Using the notations of sec. 2.2, it is easy to find that the appropriate form is

$$
G=U_{v} \int_{0}^{\infty} \rho_{f}^{\text {cross }}(x, t) \frac{x^{2}}{a^{2}} d x
$$

(where the time $t$ in the integral is chosen after completion of the reaction). This expression appears to be similar to eq 0 for the extent of mixing. Thus, we conclude that in this case $(\alpha \gg 1)$, the zero-rate adhesion energy $G$ would be simply proportional to the final extent of 


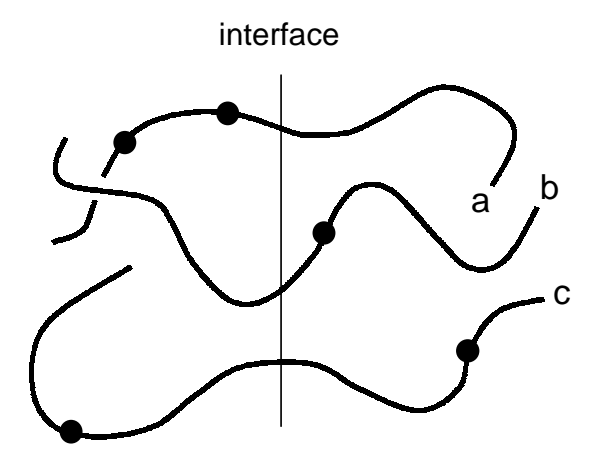

Figure 7: Chains at the interface in the final state of the slow reaction regime. Most chains have arms of comparable size on both sides of the interface. Upon opening of a fracture, chain 'a' will pull its right-side portion out, chain 'b' will pull its left-side portion out, and chain 'c' will undergo a scission process.

mixing:

$$
G \simeq U_{v} \Gamma_{f}^{\text {final }}
$$

(with $\Gamma_{f}^{\text {final }}$ given by eq 17 or 18 ).

We now consider the slow reaction regime $(\alpha \ll 1)$. In this case, as already stated, the interface heals before the reaction has significantly advanced, so that most interfacial chains spread arms of comparable size $(\sim N)$ on both sides of the junction by the time they are pinned down. On Figure 7, which presents some possible configurations of the interfacial chains, we see that in addition to the chains that will have to be pulled out if we try to open a fracture, some chains are anchored on both sides and will rather undergo a scission. (The contribution of such scission processes to the adhesion energy $G$ is estimated in the Appendix.) If we assume that the scission and the pull-out contribution simply add to each other, we find that, again, $G$ is related to the final extent of mixing $\Gamma_{f}^{\text {final }}$ by a simple proportionality, in a similar way to eq 22 :

$$
G \simeq U_{0} \Gamma_{f}^{f i n a l}
$$

where $U_{0}$ is an energy, usually comparable to that of a chemical bond, and $\Gamma_{f}^{f i n a l}$ should be taken as in eq 19). A derivation of this result is available in the Appendix.

Let us now consider the molecular weight dependence of these (zero-rate) adhesion energies in the fast and slow reaction regimes. In the fast reaction regime, we have $G \simeq U_{v} \Gamma_{f}^{f i n a l}$ (eq 22) with $\Gamma_{f}^{f i n a l}$ as given by eq 17 or 18 . The dependence in $N$ of the parameter $\alpha=Q \tau_{0} A_{0}^{*} N^{3} b^{3} / N_{e}$ can be evaluated as follows: in situations where the initial concentration $A_{0}^{*}$ is a finite, $N$ independent, fraction of the total monomer concentration $\left(A_{0}^{*}=p / a^{3}, p<1\right)$, we have $\alpha \sim N^{3}$. 
Alternately, if there are only a few $A^{*}$ sites on each chain $\left(A_{0}^{*} \simeq 1 / N a^{3}\right)$, we have $\alpha \sim N^{2}$. Together with $\rho_{0} \sim 1 / N, R_{0} \sim N^{1 / 2}$, we get

$$
G_{\text {fast }} \sim \begin{cases}N^{-7 / 4} & \left(A_{0}^{*}=p / a^{3}\right) \\ N^{-1} & \left(A_{0}^{*} \simeq 1 / N a^{3}\right) .\end{cases}
$$

On the other hand, it is easy to estimate the $N$-dependence of the slow reaction regime, by using $G \simeq U_{0} \Gamma_{f}^{f i n a l}\left(\right.$ eq 23) and $\Gamma_{f}^{\text {final }} \simeq \rho_{0} R_{0}^{3} / a^{2}$ (eq 19):

$$
G_{\text {slow }} \sim N^{1 / 2}
$$

It appears that the fast and slow reaction regimes exhibit clearly distinct dependences on the molecular weight of the polymer: $G_{\text {fast }}$ has a marked inverse dependence on $N$, whereas $G_{\text {slow }}$ displays a square-root, increasing, dependence. Such predictions may prove testable on the experimental side, either by direct macroscopic measurements of the adhesion strength, or by microscopic techniques surveying the local evolution of the interpenetration (one would then rather have access to the extent of mixing $\Gamma_{f}^{\text {final }}$, but molecular weight dependences are identical).

In our approach, the adhesion energy $G$ is proportional to the final extent of mixing $\Gamma_{f}^{f i n a l}$ (obtained after completion of the crosslinking reaction). Since $\Gamma_{f}^{f i n a l}$ saturates when the interpenetration distance is of the order of the Gaussian size $R_{0}$ of the chains (see section 2.2), $G$ saturates to its maximum value at the same time. This result is related to the fact that our systems are maintained above the glass transition (allowing relative sliding of chains), and that we are dealing with adhesion energies at low separation velocities. The property that the full tensile strength of a latex film is established over a time comparable to, or larger than, the reptation time is supported by several experimental studies (see ref. [6] and references therein). We emphasize, however, that glassy polymers may display rather different features [25].

We conclude this section by stressing that all these estimations of the adhesion work remain of course very crude, but they suggest a simple link between a microscopic quantity like the extent of mixing and the macroscopic adhesion energy.

\subsection{Other systems}

In the previous sections, we considered a system where the strenghtening of the interface occurs in the presence of a crosslinking agent. When the system is optimized, chains have time to diffuse across the interfaces before being locked by the chemical reaction and form permanent bridges. This strategy is not the only available, and we will here briefly consider a few other approaches of use in the latex film technology.

1) One possibility is to use a copolymer containing as one of the comonomers a functionality $Y$ which, upon addition of a catalyst, is able to react with another $Y$ to form a dimer. This 


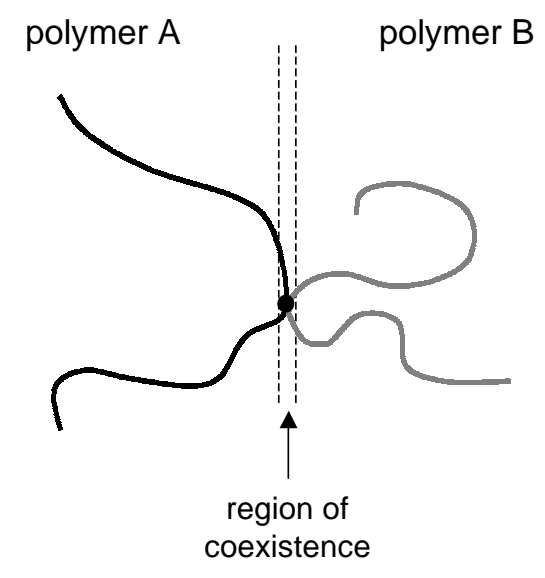

Figure 8: Block copolymer formed by reaction at the border region between two incompatible polymers. Chains from each kind remain confined to their own particle, but the formation of crosslinks in the coexistence region creates copolymer bridges that will have to be pulled out if a fracture opens.

reaction leads to the formation of $Y-Y$ bridges between polymer chains, throughout the system, at the interfaces and in the bulk of the polymeric material. We can see that this is very similar to our system with a crosslinker agent, as soon as we neglect the first attachment of the crosslinker to the macromolecules.

2) A second possibility is to use a mixture of two different kinds of particles, for instance one sort made of a polymer A, and the other made of a polymer B, where chains of polymer A bear sites able to react with sites of polymer B. We may further assume that polymer A and B are strongly immiscible $(\chi>0$ and $\chi N \gg 1$, with $\chi$ the associated Flory parameter), so that the region where $\mathrm{A}$ and $\mathrm{B}$ coexist is very thin compared to the chain radius. In this case, $\mathrm{A}$ and $\mathrm{B}$ chains react only within the interface, and form block copolymers at the junction (see Figure 8). Theory actually predicts a host of different chemical kinetics regimes for the formation of such bridges [26, 27, 28]. There is, in the present situation, a significant strengthening of the interface, even though the extent of mixing remains vanishingly small: as seen on Figure 8, here again, one must pull out the interfacial copolymers out of their surrounding matrix to open a fracture. To characterize the strength of the interface, the adhesion energy formula of eq 20 discussed above seems suitable, and we may use it to give a few guidelines to optimize A/B systems. From eq 20 , we see that we need the longest possible attached strands at the interface: this would suggest to have only one reactive site per chain (for $\mathrm{A}$ as well as for $\mathrm{B}$ ). We would also wish to reach the highest possible interface coverage by connectors $\left(\sigma \simeq \rho_{0} R_{0}\right)$, a condition which should be 
easy to fulfill, because it is a natural tendency of the interfacial reaction [26, 27, 28]. Then the interfacial adhesion energy would be of order

$$
G \simeq U_{0} \frac{1}{a^{2} N^{1 / 2}} N \simeq G_{\max }
$$

similar to the value reached with crosslinked systems.

3) Another variant of the A/B system is the case of a miscible pair A/B. This situation, however, raises difficulties of its own: reactions occur in a non-stationary, spreading region, making the computation of reactional quantities very difficult. In the case of small molecules with a time-dependent reaction front, theoretical studies [29, 30] predict anomalous laws for reaction rates. But, to our best knowledge, the macromolecule case (of interest to us) still remains to be clarified.

\section{Acknowledgements}

It is a pleasure to thank Prof. M.A. Winnik of the University of Toronto, who introduced us to this subject, and helped us forward with fruitful exchanges. The authors are also thankful to C. Creton, L. Léger, and the anonymous referees, for discussions and useful comments.

\section{Appendix. Estimation of the adhesion energy in the slow reaction regime}

In this Appendix, we want to estimate the adhesion energy between the particles in the final state of the slow reaction regime (Figure 7).

We start by evaluating the density of connectors chains (crossing chains) $\sigma_{f}$ at the end of the reaction. If we consider an area $\mathrm{S}$ of interface, the chains crossing it must lie within a distance $R_{0}$, so that there is a number $\rho_{0} R_{0} S$ of them. This corresponds to a grafting density $\sigma_{f} \simeq \rho_{0} R_{0}$.

A fraction $f_{1}$ of these crossing chains have reacted only on one side of the interface, and thus contribute to the adhesion by a pull-out mechanism. Considering that most crossing chains

display arms of comparable size $\sim N$ on both sides of the interface, and using eq 20 to compute the corresponding adhesion energy, we find a contribution $G_{\text {pull-out }} \simeq U_{v} f_{1} \sigma_{f} N$.

But a fraction $f_{2}$ of the interfacial chains is anchored on both sides of the interface (Figure 7), and rather than being pulled out, they undergo a scission when the interfacial fracture opens. For this scission mechanism, we may evaluate the adhesion energy using the classical Lake and Thomas argument [31]: at the moment of rupture each monomer along one connector chain has stored an energy comparable to the chemical bond energy $U_{\chi}$. Hence, to bring a chain to rupture, we have to provide an energy $U_{\chi}$ to all the monomers between the anchorage points. 
Since crosslinked points are not too numerous on each chain, in average the number of monomers under tension is $\sim N$ (within factors). We may then evaluate the scission contribution to the adhesion energy as $G_{\text {scission }} \simeq U_{\chi} f_{2} \sigma_{f} N$.

We note that $G_{\text {pullout }}$ and $G_{\text {scission }}$ have the same structure (at this scaling law level). If we assume that these energies add to each other into a global adhesion energy $G_{t o t}$, we have $G_{t o t} \simeq U_{0} \sigma_{f} N$, where the energy $U_{0}$ would be a weighted average of $U_{\chi}$ and $U_{v}$ with respect to the probabilities of the scission and pull-out mechanisms. Then, using $\sigma_{f}=\rho_{0} R_{0}$ and $N=R_{0}^{2} / a^{2}$, we see that this adhesion energy is related to the final extent of mixing (eq 19) in a proportional way:

$$
G_{\text {tot }} \simeq U_{0} \rho_{0} R_{0} R_{0}^{2} / a^{2} \simeq U_{0} \Gamma_{f}^{\text {final }},
$$

as stated in section 4.2. We add that, because the covalent energy $U_{\chi}$ is much larger than the van der Waals energy $U_{v}\left(U_{\chi} / U_{v} \simeq 40\right), U_{0}$ should be, in many cases, dominated by the scission contribution, or equivalently, $G_{t o t} \simeq G_{\text {scission }}$.

\section{References}

[1] See: Wool, R.P. Polymer Interfaces: Structure and Strength; Hanser Publications: Cincinnati, 1995 and references therein. See also: Jones, R.A.L; Richards, R.W. Polymers at Surfaces and Interfaces; Cambridge University Press: Cambridge, 1999.

[2] de Gennes, P.-G. C. R. Acad. Sci. Paris, Ser. B 1980, 291, 219.

[3] de Gennes, P.-G. In Physics of Polymer Surfaces and Interfaces; Sanchez, I.C., Fitzpatrick, L.E., Eds.; Butterworth-Heinemann; Manning Publications Co.: London, 1992; p 55.

[4] Prager, S.; Tirrell, M. J. Chem. Phys. 1981, 75, 5194.

[5] Kim, Y.H.; Wool, R.P. Macromolecules 1983, 16, 1115.

[6] Winnik, M.A. Curr. Opinion Coll. Interf. Sci. 1997, 2, 192.

[7] Taylor, J.W.; Winnik, M.A. "Functional Latex and Thermoset Latex Films". In Functional Colloids and Fine Particles; Guyot, A., Ed.; Citus Books: London, 2000.

[8] Bufkin, B.G.; Grawe, J.R. J. Coatings Technology 1978, 50, No. 641, 41.

[9] Feng, J.; Pham, H.; Macdonald P.; Winnik, M.A.; Geurts, J.M.; Zirkzee, H.; van Es, S.; German, A.L. J. Coatings Technology 1998, 70, No. 881, 57.

[10] Tamai, T.; Pinenq, P.; Winnik, M.A. Macromolecules 1999, 32, 6102. 
[11] de Gennes, P.-G. J. Phys. 1975, 36, 1199.

[12] To be more specific, in the context of filmification from a dispersion of latex particles in water, the crosslinker agent is originally in the aqueous solution, and diffuses inside the polymer particles. We suppose in the text that this diffusion inside the polymer phases and the subsequent attachment reaction (our so-called "first step") are fast processes (compared to the polymer reactions), so that they are completed during the evaporation of the solvent, or very quickly after onset of contacts. Even further, in many real cases, not only the first step is finished at the time of contact, but the polymer-polymer reaction step has already begun inside individual particles, in the dispersed state. Such a premature crosslinking reduces the amount of mobile chains that will be available for interdiffusion when the particles come in contact (at $t=0$ ).

[13] Chang, R.-J.; Gent, A.N. J. Polym. Sci.: Polym. Phys. Ed. 1981, 19, 1619.

[14] Gent, A.N.; Lai, S.-M. J. Polym. Sci.: Polym. Phys. Ed. 1994, 32, 1543.

[15] This statement is, in fact, somewhat inaccurate: when a site reacts, the number of chains that stop as a consequence depends on the nature of the partners of the reaction. If the reaction occurred between one $A^{*} X$ borne by a mobile chain and a $A^{*}$ borne by another mobile chain, then for a single reaction, two chains are stopped. If the reaction occurs between a mobile chain and a fixed one, then only one chain stops, and zero when it occurs between two fixed chains. However, one can show that these subtleties are not very significant at our scaling law level.

[16] de Gennes P.-G. J. Chem. Phys. 1971, 55, 572.

[17] Doi, M.; Edwards, S.F. The Theory of Polymer Dynamics; Clarendon Press: Oxford, 1986.

[18] de Gennes, P.-G. J. Chem. Phys. 1982, 76, 3316.

de Gennes, P.-G. J. Chem. Phys. 1982, 76, 3322.

[19] O’Shaughnessy, B.; Vavylonis, D. Europhys. Lett. 1999, 45, 638.

[20] O'Shaughnessy, B. Macromolecules 1994, 27, 3875.

[21] O'Shaughnessy, B.; Vavylonis, D. Eur. Phys. J. E 2000, 1, 159 and references therein.

[22] Wang, Y.; Winnik, M.A. J. Phys. Chem. 1993, 97, 2507.

[23] Pham, H.H.; Winnik, M.A. Macromolecules 1999, 32, 7692.

[24] Raphaël, E.; de Gennes P.-G. J. Phys. Chem. 1992, 96, 4002. 
[25] It has been shown by Schnell et al. (Schnell, R.; Stamm, M.; Creton, C. Macromolecules 1998, 31, 2284) that when two glassy samples of a high molecular weight polymer are put into contact and heated above the glass transition during a limited amount of time, so as to allow for a controlled amount of interdiffusion across the junction, the maximum of the adhesion strength is reached when the interpenetration has occurred over a distance comparable to the average length between entanglements (i.e., much earlier than the reptation time). This property highlights the important role of entanglements in the development of adhesion in glassy polymers.

[26] Fredrickson, G.H.; Milner, S.T. Macromolecules 1996, 29, 7386.

[27] O’Shaughnessy, B.; Sawhney, U. Macromolecules 1996, 29, 7230.

[28] O’Shaughnessy, B.; Vavylonis, D. Macromolecules 1999, 32, 1785.

[29] Gálfi, L.; Rácz, Z. Phys. Rev. A 1988, 38, 3151.

[30] Lee, B.P.; Cardy J. Phys. Rev. E 1994, 50, R3287.

[31] Lake, G.J.; Thomas, A.G. Proc. R. Soc. London Ser. A 1967, 300, 108. 